\title{
A case of Plasmodium falciparum malaria: the importance of an integrated diagnostics
}

\author{
Paola Notaris, Lorena Montevecchi, Ernesto Delprete \\ Servizio Unificato Laboratorio Analisi Zona Territoriale N.3 di Fano - ASUR Marche
}

Key words: P. falciparum, Thin blood smear, Immunochromatography assay

\section{Un caso di malaria da Plasmodium falciparum: l'importanza di una diagnostica integrata}

\section{SUMMARY}

We report a case of Plasmodium falciparum in an Italian woman of thirty years old, back from Mozambico. The diagnostic suspect came from various factors: a recent stay in a country where the disease is endemic, fever and an inadeguate prophylaxis. The presence of Plasmodium falciparum in thin blood smear and in the immunochromatography assay positivity confirmed the diagnosis.

\section{INTRODUZIONE}

La malaria è forse la più importante malattia parassitaria dell'uomo e tra le principali cause di morbosità e mortalità in particolar modo nelle aree tropicali e subtropicali, ove la medesima è endemica, senza dimenticare che anche i Paesi indenni da sempre o in cui la malattia è stata da tempo eradicata devono registrare ogni anno migliaia di casi di importazione, dovuti in particolare all'aumento esponenziale dei viaggi internazionali ed all'intensificazione del fenomeno migratorio (17).

Anche in Italia, come in paesi storicamente legati ai continenti ad elevata endemia malarica (Regno Unito, Francia, Olanda, Belgio e Portogallo), si è avuto un aumento dei casi di malaria di importazione. Si è registrato un costante aumento dal 1970 al '78, un lieve calo dal 1979 all' 84 e un allarmante aumento tra il 1987 e l'89, così come nei periodi 1990-95 e 1996-2000, sino ad arrivare a circa 1000 casi nell'anno 1999. A partire dal 1995 il numero dei casi rilevati negli stranieri ha superato quello dei casi di cittadini italiani (7); l'aumento è dovuto allo sviluppo di flussi immigratori dai paesi africani Sub Sahariani verso l'Italia (15).

L'Italia è stata dichiarata indenne da malaria dall'OMS nel 1970. Gli ultimi casi furono infatti osservati nei primi anni Sessanta. Da allora la quasi totalità dei casi notificati sono di importazione. Secondo quanto riportato dall'Istituto Superiore di Sanità, ogni anno vengono osservati in Italia circa 600 casi con un numero di decessi che nel periodo 2002-2006 era compreso tra 1 e 4 per anno (16). L'agente causale è il plasmodio, un protozoo trasmesso con la puntura di zanzare fem- mine del genere Anopheles. Ad ammalarsi sono principalmente gli immigrati che tornano al paese d'origine per visitare parenti o amici o gli italiani che per diversi motivi (volontariato, lavoro, turismo) visitano le zone a rischio. È importante sottolineare che la profilassi e la terapia antimalarica rappresentano un problema di Sanità Pubblica in considerazione del fenomeno della farmaco-resistenza dei ceppi di Plasmodium falciparum (3). Negli ultimi anni vi sono stati inoltre rarissimi casi di malaria dovuti a modalità di trasmissione inconsueta come trapianto d'organo, trasfusione di sangue, punture accidentali con materiale contaminato e, infine, casi "criptici" (dovuti probabilmente a zanzare trasportate con il bagaglio). Secondo i dati di un recente studio condotto da ricercatori dell'I.S.S. (Istituto Superiore di Sanità) e del Ministero della Salute (pubblicato sul "Giornale italiano di medicina tropicale", vol.12, n. 1- 4, 2007), si conferma la tendenza verso una riduzione dei casi importati, con una percentuale dei decessi stabile e inferiore alla media europea (11). L'ultimo caso sporadico di malaria autoctono (plasmodio di importazione, ma trasmesso da una zanzara Anopheles "autoctona"), risale al 1997 e si è verificato nella provincia di Grosseto, dove una signora è stata infettata da $P$. vivax tramite una zanzara che aveva precedentemente punto una bambina indiana affetta da malaria (16).

La diagnosi di infezione, partendo dal sospetto anamnestico - clinico, è basata sulla capacità di rilevare l'agente e i suoi antigeni a livello ematico. Pur essendo una condizione a bassa prevalenza, la pericolosità e la rapida evoluzione dell'infezione rendono necessario predisporre e mantenere

\section{Corresponding author: Paola Notaris}

Servizio Unificato Laboratorio Analisi Zona Territoriale N.3 Fano (PU)

Viale V. Veneto 2; 61032 Fano (PU) - Tel.072I 882379 / 732765 - Fax 072 I 882304

E-mail: paola.notaris@sanita.marche.it 
disponibili competenze professionali e metodologie diagnostiche che permettono un rapido riconoscimento o esclusione della patologia. Si tratta quindi di un importante banco di prova per il microbiologo che deve prepararsi ed essere reperibile 24 ore su 24.

\section{Descrizione del caso clinico}

Z. B. è una donna italiana di trenta anni, rientrata da una settimana dal Mozambico dopo un mese di permanenza a seguito di una missione umanitaria. Si è presentata al Pronto Soccorso dell'Ospedale di Fano un giorno festivo del maggio 2010 con iperpiressia accompagnata a brivido e artromialgie (dolori addominali, cefalea e vomito erano assenti). La paziente aveva fatto profilassi antimalarica con farmaci omeopatici in quanto risultata intollerante alla Meflochina, farmaco antimalarico utilizzato in un viaggio precedente. L'esame obiettivo del medico di guardia del Pronto Soccorso evidenzia una paziente eupnoica con febbre a $39.2^{\circ} \mathrm{C}$ senza segni clinici indicativi di anemizzazione acuta in atto, addome trattabile non dolente alla palpazione, non organomegalie. Al torace non rumori aggiunti. Dall'esame obiettivo della paziente i parametri clinici risultano normali: frequenza cardiaca $120 / \mathrm{min}$, pressione arteriosa $120 / 80 \mathrm{~mm} / \mathrm{Hg}$, saturazione $\mathrm{O}_{2} 94 \%$.

Vengono richiesti diversi esami emato-chimici e la ricerca del plasmodio malarico. Dagli accertamenti emato-chimici il dato più rilevante è rappresentato dalla piastrinopenia $107.000 / \mathrm{mmc}$, e da un'alterazione dei valori coagulativi D-dimero $1007 \mathrm{ng} / \mathrm{ml}$. Nel nostro laboratorio l'identificazione del parassita malarico è condotta integrando la ricerca immunocromatografica degli antigeni malarici con l'osservazione microscopica del sangue periferico.

Contemporaneamente venivano preparati dei vetrini per la diagnosi microscopica, tenendo presente che una corretta preparazione dello striscio permette di determinare parassitemie anche molto basse (6). I vetrini uniformi e sottili venivano lasciati asciugare all'aria per diversi minuti e colorati con Wright-Giemsa utilizzando un coloratore automatico (il manuale operativo di procedure standard del WHO consiglia l'uso della colorazione di Giemsa) (1).

La ricerca è stata effettuata per un numero superiore ai 100 campi con obiettivo $63 \mathrm{x}$, mentre è stato utilizzato quello a 100x per la conferma quando veniva riscontrato un trofozoite. Erano presenti trofozoiti con citoplasma ad anello (ringform) (Figura I).

Una diagnosi rapida e accurata rappresenta il presupposto indispensabile per una terapia efficace nei soggetti affetti da malaria. Confermata quindi l'ipotesi di malaria sia con il test rapido che con lo striscio di sangue periferico, la paziente, nello stesso giorno veniva inviata al reparto di Malattie Infettive presso l'Ospedale di Pesaro dove le è stata somministrata immediatamente la terapia.

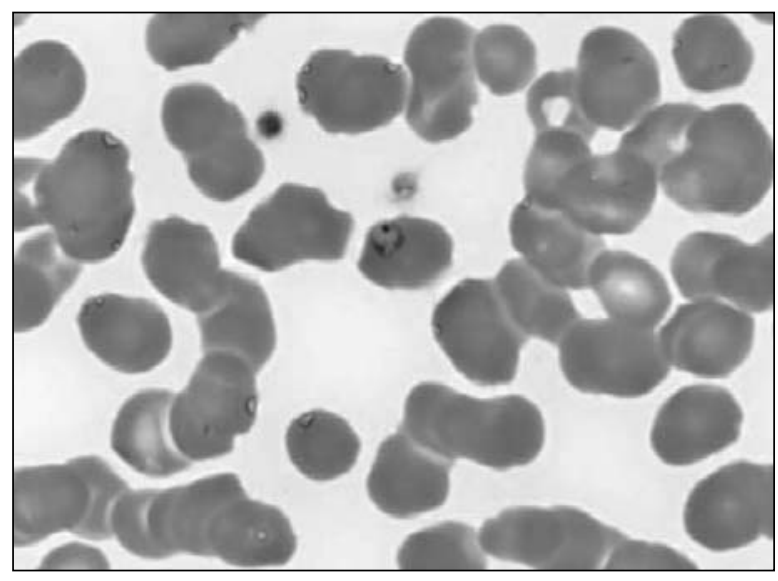

Figura I. Tipico trofozoite di Plasmodium falciparum ritrovato all'esame dello striscio sottile del caso riportato.

\section{DISCUSSIONE}

La ricerca del parassita malarico si impone al minimo sospetto anamnestico e/o clinico come ad esempio un paziente che rientra da una zona endemica con sintomatologia simil-influenzale (febbre, astenia, disturbi gastrointestinali, artromialgie, cefalea) e va eseguita anche se il paziente non presenta febbre ed ha effettuato una chemioprofilassi. Nel nostro caso, l'anamnesi del viaggio in una regione di endemia malarica, la mancata profilassi o meglio una profilassi eseguita con farmaci omeopatici (del tutto inadeguata e decisamente non idonea) hanno fatto porre il sospetto diagnostico di malaria al medico del Pronto Soccorso che ha immediatamente richiesto al laboratorio analisi la ricerca dei parassiti sia su striscio di sangue periferico sia su test rapido. Il riscontro dei parassiti all'esame dello striscio di sangue è stato diagnostico di malaria.

L'antigene positivo per $P$. falciparum al test rapido ha permesso di sospettare l'infezione e di confermare la presenza di P. falciparum.

Il quadro clinico varia, sotto alcuni aspetti, in rapporto al plasmodio in causa; nello specifico, le infezioni da $P$. falciparum rappresentano una vera e propria emergenza medica, in particolare in quei soggetti non immuni dove la malattia si caratterizza per un decorso generalmente grave o molto grave (dovuto alla marcata citoaderenza degli eritrociti infetti) (18). Secondo l'OMS la malaria è una malattia curabile se la diagnosi è tempestiva e se la scelta della terapia tiene conto della zona di provenienza (4). La ricerca del parassita è quindi da considerarsi a tutti gli effetti un esame d'urgen- 
za. La malaria è infatti la sola reale urgenza nell'ambito delle malattie parassitologiche perché in 12-24 ore un soggetto non immune può passare da una discreta condizione psicofisica ad uno stadio di coma per malaria cerebrale da Plasmodium falciparum.

Nella nostra piccola realtà, questo caso di malaria, che poteva essere grave, è stato gestito correttamente e in tempi accettabili grazie ad una tempestiva diagnosi. La paziente infatti, è stata trasferita, dopo poche ore di degenza presso il Pronto Soccorso al reparto di Malattie Infettive. Il laboratorio analisi ha risposto rapidamente alle richieste del Pronto Soccorso inviando in prima battuta, e in tempi molto rapidi, il referto degli esami ematochimici e del test rapido, mentre si è reso necessario utilizzare un tempo maggiore (circa $2 / 3$ ore) per la lettura dello striscio di sangue a causa del basso indice di parassitemia (I.P. $=0.007 \%$ ). In caso di negatività sarebbe stato utile ripetere l'esame emoscopico dopo 6-8 ore o comunque alla successiva puntata febbrile.

I vantaggi del test rapido sono dovuti alla standardizzazione della metodica, ad una discreta specificità $(94.2 \%$ con un intervallo di confidenza del 93-95\%) e ad una buona sensibilità quando il numero dei parassiti per $\mu 1$ di sangue è compreso tra 100 e 500 o con valori superiori $(8,9,19)$.

Come si evince dalla Tabella 1, il limite del test rapido è legato al decremento della sensibilità a bassi livelli di parassitemia, in particolare quando il livello si attesta tra 0 e 100 per $\mu 1$. Nel nostro caso abbiamo calcolato una parassitemia dello $0.007 \%$ e un numero di parassiti per $\mu l$ di sangue pari a circa 371 parassiti $/ \mu 1$; (I.P. $=$ G.R. parassitati su 1000 e riportare in percentuale) (4-19) [usando l'obiettivo ad immersione 63x (obiettivo Olympus UPlan FLN 60x/1.25 oil immersion) in cui un campo microscopico, contiene mediamente 550 emazie]. Il test inoltre non permette di effettuare una diagnosi certa di specie, potendo distinguere esclusivamente la malaria da Plasmodium falciparum, da quella non falciparum e non è stata adeguatamente stabilita l'efficacia clinica per $P$. ovale e $P$. malariae. Nella diagnosi di malaria, questo test può risultare un ausilio diagnostico particolarmente utile in caso di infezioni con bassa parassitemia o in situazioni di urgenza, quando è importante conoscere rapidamente se un caso di malaria diagnosticato clinicamente è dovuto a falciparum o ad altre forme plasmodiali. Inoltre presenta diversi vantaggi: non richiede microscopio o altra apparecchiatura, sono necessari pochi minuti per il risultato (tempo massimo di lettura entro 15 minuti), ha un controllo positivo integrato per svelare eventuali errori di procedimento. Proprio per la sua bassa sensibilità la ricerca dell'antigene non può essere refertata senza aver fatto anche l'esame emoscopico con striscio e goccia spessa, come prevedono le norme vigenti. Il dosaggio immunocromatografico (BinaxNOW Malaria) per la determinazione qualitativa degli antigeni di Plasmodium spp. è un test rapido in vitro che utilizza sangue intero trattato con EDTA.

Il test ha come bersaglio l'antigene solubile HRPII (specifico per $P$. falciparum e costituito da una glicoproteina ricca di istidina che viene liberata durante il ciclo eritrocitario del parassita con un picco durante la rottura degli schizonti) e l'antigene panmalarico (antigene comune a tutte le specie di Plasmodium che causano la malattia nell'uomo: $P$. falciparum, $P$. vivax, $P$. ovale e $P$. malariae). Esistono in commercio alcuni test che rilevano tre antigeni e che permettono una discreta specificità per l'identificazione di specie sia di P. falciparum, che per $P$. vivax e $P$. ovale anche se non distingue tra le due e per $P$. malariae.

Vanno infine fatti notare i ruoli del fattore reumatoide e della persistenza dell'antigene, che può essere rilevato anche dopo diversi giorni dall'eliminazione del parassita, quali cause di false positività (11); ciò rende il test inadatto al monitoraggio del trattamento della malaria.

Allo stesso tempo un test negativo non esclude la malattia, in particolare a bassi livelli di parassitemia. La piastrinopenia in caso di sospetto di malaria ha una sensibilità dell' $80 \%$, non molto lontana dalla sensibilità dell'antigene in caso di bassa parassitemia. Dallo striscio ematico è invece possibile ricavare:

1) la diagnosi di specie;

2) il calcolo della parassitemia;

3) la morfologia delle emazie (valutazione per eventuale anemia falciforme associata) e riconoscere altre alterazioni a carico dei globuli

Tabella I. Valutazione del kit BinaxNow Malaria per P. falciparum

\begin{tabular}{ccc}
\hline Livelli di concentrazione per $\mu \mathrm{l}$ & \% Sensibilità & Intervallo di confidenza \% \\
\hline & 99.7 & $98-100$ \\
\hline $1000-5000$ & 99.2 & $96-100$ \\
\hline $500-1000$ & 92.6 & $76-99$ \\
\hline $100-500$ & 89.2 & $75-97$ \\
\hline $0-100$ & 53.9 & $37-70$ \\
\hline Totale & $\mathbf{9 5 . 3}$ & $\mathbf{9 3 - 9 7}$ \\
\hline
\end{tabular}


rossi o delle altre linee cellulari (10).

Anche l'esame emoscopico presenta limiti di sensibilità; le alte parassitemie non sfuggono alla diagnostica tradizionale, mentre il problema si pone nei casi di bassa o bassissima parassitemia che può essere presente in tre situazioni cliniche:

1) soggetti semi-immuni che sviluppando un'importante reazione immunitaria impediscono una schizogonia massiva;

2) infezione malarica in soggetti non immuni in corso di profilassi, o in seguito a profilassi impropria o incompleta;

3) infezioni da P. falciparum molto sincronizzate; questi ultimi sono casi rari in cui lo striscio può essere negativo (parassitemia al di sotto della soglia) in quanto quasi tutti i plasmodi sono allo stadio di schizonte, sequestrati nei capillari degli organi interni e quindi assenti nel sangue periferico, oppure perché vi è stata emolisi massiva dovuta alla rottura degli schizonti stessi e dell'emazia che li conteneva (11). Le modalità di allestimento dei vetrini possono comprendere lo striscio sottile e la goccia spessa con colorazione con acqua tamponata a $\mathrm{pH} 7.2 \mathrm{e}$ Giemsa. May-Grunwald-Giemsa può essere utilizzato solo per lo striscio perché fissa la goccia spessa che non deve essere fissata. Lo striscio sottile è da preferire per l'accuratezza, poiché consente di apprezzare le differenze di specie tra $\mathrm{i}$ vari plasmodi, mentre la goccia spessa, molto utile in caso di bassa parassitemia, non permette un esame comparativo, ma un semplice riscontro della presenza generica di trofozoiti; ha maggior sensibilità poiché permette l'esame di una maggior quantità di sangue (circa 20 volte), ma al contempo l'esame diventa più difficoltoso in mano ai laboratoristi che non eseguono questo esame di routine; lo striscio sottile, invece, è una metodica di facile preparazione e alla portata di tutti i laboratori, purché si sia già visto qualche preparato positivo e qualche altro negativo con alcuni artefatti che potrebbero indurre in false positività (es.: piastrine). Perché il test abbia più probabilità di successo è opportuno eseguire lo striscio in occasione degli accessi febbrili quando è più probabile avere trofozoiti in circolo. L'esame va eseguito anche in assenza di febbre data la sua urgenza e se negativo andrà ripetuto al successivo attacco febbrile. La sensibilità che un esperto microscopista può raggiungere sui preparati con goccia spessa è di circa $10-50$ parassiti/ $\mu$ che equivale ad un I.P. dello $0.001 \%$ (19). Tuttavia lo stesso lavoro riporta $\mathrm{i}$ risultati provenienti dalla maggior parte dei laboratori britannici non specializzati che fanno capo al "Centro di riferimento per la malaria" dai quali si evince che la sensibilità da questi raggiunta è significativamente inferiore, con un I.P. che si attesta attorno allo $0.01 \%$ con circa 500 parassiti/ $\mu 1$ di sangue (13). La presenza del parassita nel nostro caso, caratterizzato da un I.P. attorno allo $0.007 \%$ (che corrisponde a 371 parassiti/ $\mu 1$ ), valore che è al di sotto della soglia di sensibilità raggiunta dai laboratori non specializzati, avrebbe potuto passare inosservata senza l'ausilio del test immunocromatografico, la cui positività ci ha stimolato ad un'approfondita e prolungata ricerca dei trofozoiti nei diversi vetrini che avevamo allestito. Va sottolineato che lo striscio va esaminato almeno per 400 campi a $100 x$ prima di essere dato negativo. Come riportato in letteratura l'efficacia del test rapido dipende dalla quantità di antigeni presenti nel campione e può non correlarsi direttamente all'esame microscopico eseguito sullo stesso campione $(8,9,19)$. L'assenza di correlazione diretta tra il test rapido e l'esame emoscopico è una caratteristica di questo caso; difatti, di fronte ad un test rapido positivo, si è contrapposto una bassa carica parassitemica osservata su striscio sottile. Va anche ricordato che questo caso è stato rilevato e diagnosticato in un giorno festivo (nel laboratorio era presente solo il personale di guardia) e in un Ospedale dove sono di raro riscontro le patologie "esotiche". L'impiego associato del test immunocromatografico e della microscopia a nostro avviso consente di raggiungere livelli soddisfacenti di specificità, sensibilità, accuratezza e tempestività della risposta anche a fronte di patologie "rare" e in condizioni di urgenza visto la loro rapida evoluzione anche infausta e di laboratori che non hanno esperienza microscopica per la diagnosi di malaria. E necessario sottolineare che ogni laboratorio deve sviluppare un minimo di esperienza in ogni persona coinvolta nelle attività di urgenza. Per quanto riguarda la malaria è necessario formare tutto il personale sull'idoneità del prelievo, le modalità di preparazione e la lettura dello striscio e della goccia spessa, la qualità del colorante, il pH 7.2 dell'acqua tamponata, valori e limiti dell'antigene.

\section{BIBLIOGRAFIA}

1. Baldari M, Tamburro A, Sabatinelli G, et al. Malaria in Maremma, Italy. Lancet 1998; 351: 1246-7.

2. Bernieri F, Crotti D, Galli D, Raglio A. Manuale illustrato di Diagnostica Parassitologica. Selecta Medica Editore, Pavia 2001.

3. Boccolini D, Romi R, D'Amato S, et al. Sorveglianza della malaria in Italia e analisi della casistica del quinquennio 2002-2006. Giornale Italiano di Medicina Tropicale 2007; 12: 5-12.

4. Cancrini G. Parassitologia medica illustrata. Lombardo Editore 1996; 81-107.

5. Cristallo AF. La medicina di laboratorio nella pratica medica. Selecta Medica 2008.

6. de Carneri I. Parassitologia generale e umana. Casa Ed. Ambrosiana 2004; 224-59. 
7. http:// www.dpd.cdc.gov/dpdx/HTLM/Frames/DiagnosticPr ocedures/body dp bloode.

8. http://binax.com/uploads/binaxnowmalaria-productinsert_2007.pdf

9. http://Www.epicentro.iss.it/problemi/malaria/epid.asp

10. http://www.medicoebambino.com/_malaria_plasmodium_incubazione_febbre_anemia

11. La Malaria. Medico e Bämbino pagine elettroniche 2005; 8 (5).

12. Methods manual for Laboratory quality control testing of malaria rapid diagnostic tests. Version six - June 2010 - WHO Global Malaria Programme (GMP), Geneva, Switzerland.

13. Milne LM, Chiodini PL, Warhurst DC. Accuracy of routine laboratory diagnosis of malaria in the United Kingdom. J Clin Pathol 1994; 47: 740-2.

14. Moody A. Rapid diagnostic tests for Malaria parasites. Clinical Microbiology Reviews, Jan 2002; 15: 66-78.

15. Murray CK, Gasser RA, Magill Alan J, Miller RS. Update on rapid diagnostic testing for Malaria. Clinical Microbiology Review, Jan 2008; 1: 97-100.
16. Romi R, Boccolini D, Majori G. Prevenzione e controllo della malaria d'importazione in Italia. I.S.S. Rapporti ISTISAN 01/29, 2001.

17. Scaglia M, Gatti S, Rondanelli EG. Parassiti e parassitosi umane, dalla clinica al laboratorio. Ed. Selecta Medica 2005; 23-58.

18. Stauffer W, Fischer PR. Diagnosis and treatment of malaria in children. Clinical Infectious Disease 2003; 37: 1340-8.

19. Tjitra E, Suprianto S, McBroom J, Currie BJ, Anstey NM. Persistent ICT Malaria P.f./P.v.. Panmalarial and HRP2 antigen reactivity after treatment of Plasmodium falciparum Malaria is associated with gametocytemia and results in false-positive diagnoses of Plasmodium vivax in convalescence. $J$ of Clin Microbiol, March 2001; 39: 1025-31.

20. WHO. Guidelines for the treatment of malaria. Switzerland 2006.

21. Zammarchi L, Bartalesi F, Strohmeyer M, Bartoloni A. La malaria. Internal and emergency Medicine. 5 (2) Ed. Springer-Verlag Italia S.r.l. - SIMI, Società Italiana di Medicina Interna. 